\title{
CARCINOFAUNA ASSOCIADA AO FITAL Caulerpa racemosa (FORSSKÅL) J. AGARDH E Bryopsis SPp. DO ARQUIPÉLAGO DE SÃo PEDRO E SÃO PAULO - BRASIL
}

\author{
Rodrigo Sávio Viana Pereira da SILVA ${ }^{* *}$ \\ 1 Universidade Federal de Pernambuco, Recife/PE, Brasil. E-mail: rsavioqui@hotmail.com \\ *Autor correspondente
}

\begin{abstract}
RESUMO. O fital é um habitat marinho que serve de abrigo e alimentação para a fauna e a flora relacionadas a ela. O Arquipélago de São Pedro e São Paulo (ASPSP) possui uma alta biodiversidade e há muito do que se conhecer e explorar. Este trabalho amplia os conhecimentos dos crustáceos, sendo o primeiro a inventariar a carcinofauna associada ao fital do ASPSP. Foi encontrado um Alfeídeo Synalpheus, o Majídeo Mithraculus forceps (A. Milne Edwards, 1875), o Grapsídeo Plagusia depressa (Fabricius, 1775), o Nanocassiope melanodactyla (A. Milne-Edwards, 1867). Exemplares do gênero Amphitoe e Cymadusa. Hyale sp. Hyale macrodactyla Stebbing, 1899 e Elasmopus sp., Elasmopus brasiliensis (Dana, 1855), Elasmopus rapax Costa, 1853, E. pectenicrus (Bate, 1862), E. spinidactylus Chevreux, 1907 e Elasmopus brasiliensis (Dana, 1855). Os Isopoda Joeropsis sp. também apareceram nas amostras. Os Tanaidacea estiveram representados com exemplares de Psamokalliapseudes granulosus, Leptochelia dubia, Calozodion sp. e Pseudosphyrapus sp. O ASPSP possui um registro de 32 espécies de Crustáceos; como presente trabalho tal lista de espécies cresce para 40, registrando mais oito novas ocorrências e dez novos gêneros que não foram possíveis de identificar em nível específico. Os Amphipoda, Isopoda e Tanaidacea citados são registrados pela primeira vez para o Arquipélago.
\end{abstract}

Palavras-Chave: Oceanografia, Crustáceos, Fital, Decapoda, Ilha Oceânica.

\begin{abstract}
The Phytal is an habitat, that it serves of shelter and feeding for the fauna and the flora related. The Archipelago of St. Peter and St. Paul (ASPSP) possesses one high biodiversity, still it has very of that if to know and to explore; This work comes to contribute with the magnifying of the knowledge of the crustaceans, being the first work to raise it the crustaceans associates to the macro seaweed of the ASPSP. They had been found one specie genus Synalpheus, the crab Mithraculus forceps, the Grapsidae Plagusia depressa, the Xanthidae Nanocassiope melanodactyla. The Order Amphipoda had been found family Amphithoidae genus Amphitoe and Cymadusa. Hyale e Hyale macrodactyla family Hyalidae and Elasmopus sp., Elasmopus rapax, E. pectenicrus, E. spinidactylus and E. brasiliensis family Melitidae. The Archipelago of St. Peter and St. Paul Rocks possesses a register of 32 species of Crustaceans; with our work that had the intention of raising the crustaceans associates the macro seaweed this list of species it increases for 40 species, more registering five new occurrences of species and ten new genus that had not been possible to identify the specific level. The cited species of Amphipoda, Isopoda and Tanaidacea are registered for the first time for the Archipelago.
\end{abstract}

Keywords: Oceanography, Crustaceans, Phytal, Decapoda, Ocean Island. 
Silva, Carcinofauna associada ao fital Caulerpa racemosa (Forsskal) J. Agardh e Bryopsis spp. do Arquipélago de São Pedro e São Paulo - Brasil.

\section{INTRODUÇÃO}

O fital é um habitat do ambiente marinho que serve de morada, abrigo e alimentação para a fauna e a flora relacionadas a ela (Masunari and Forneris, 1981). O termo 'fital'- do grego phyton, planta - foi proposto para designar uma terceira divisão do ambiente marinho, ao lado do pelagial e do bental, baseado no fato de que os crescimentos vegetais condicionam uma fauna própria, constante e independente do substrato (Friedrich, 1964).

Os ecossistemas fitais se caracterizam por serem uma zona de grande produção e transferência energética, devido à complexa teia trófica que naturalmente se estabelece quando tão diversificados grupos coabitam uma região fértil (Hargeman, 1966). A presença da vegetação aumenta o espaço habitável e a complexidade física da área, promovendo proteção e substrato para uma grande variedade de outras plantas, principalmente algas epífitas e animais (Alves, 1991).

É de grande interesse científico analisar qualitativamente a macrofauna associada ao fital do local por seu isolamento geográfico e importância ecológica devido à sua elevada biodiversidade.

O presente estudo vem contribuir com as pesquisas realizadas no Arquipélago de São Pedro e São Paulo (Fig. 1), objetivando caracterizar a carcinofauna associada às macroalgas do local. 


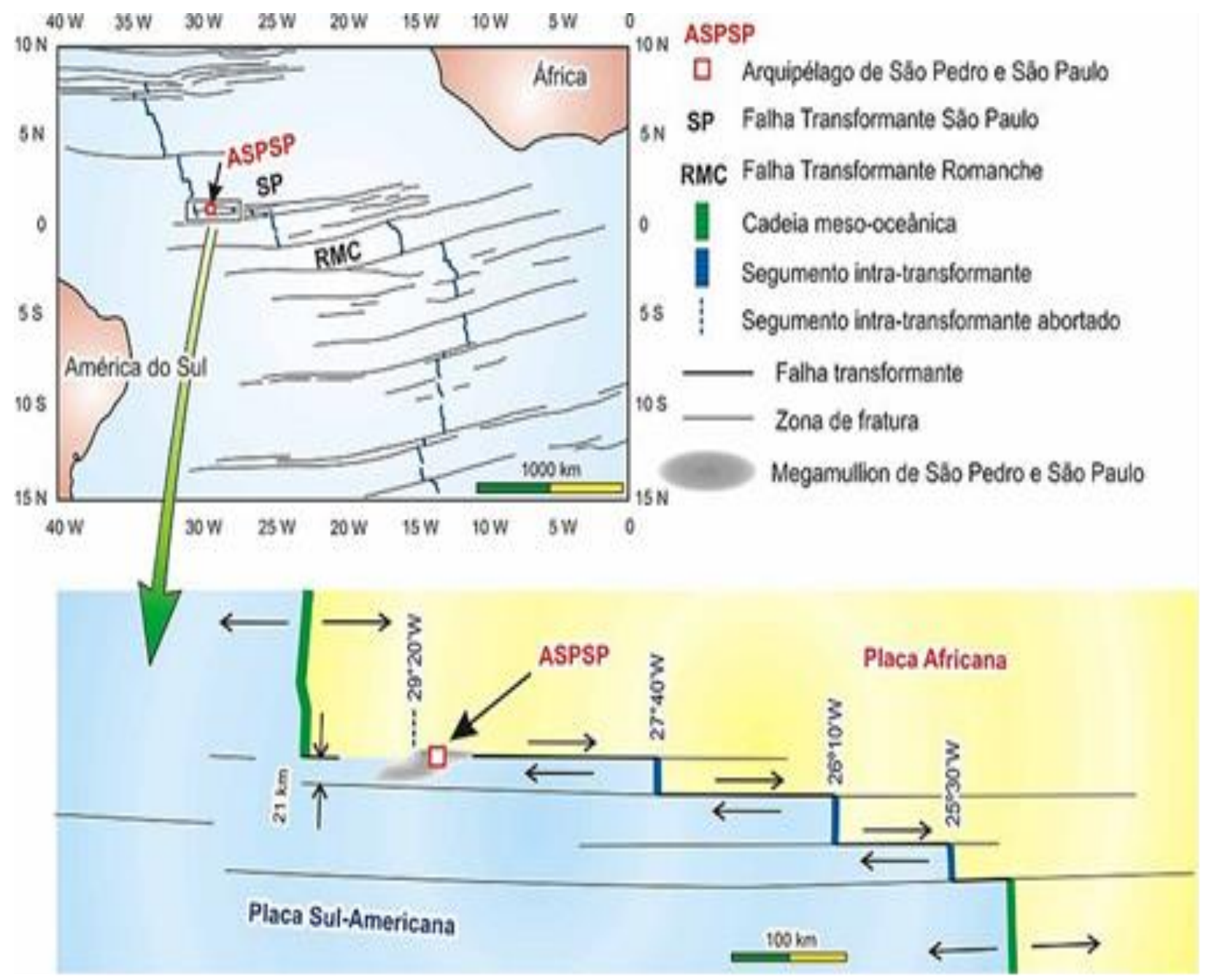

Figura 1. Localização da área de estudo $00^{\circ} 56^{\prime} \mathrm{N} ; 29^{\circ} 22^{\prime} \mathrm{W}$.

\section{MATERIAL E MÉTODOS}

O material foi procedente de amostras coligidas no Arquipélago de São Pedro e São Paulo em expedições realizadas em janeiro de 2003, maio e novembro de 2004. As plantas foram coletadas aleatoriamente numa estação fixa no fundo da enseada (Fig. 2), através de mergulho numa profundidade de $7 \mathrm{~m}$, e na parte do costão, próximo à superfície da água que sofre ação das ondas na maré baixa. Amostras de Caulerpa racemosa Forsskål no fundo da enseada foram obtidas nas três expedições, e no costão, em novembro de 2004. Tufos de Bryopsis pennata Lamouroux e B. plumosa (Hudson) C. Agardh foram recolhidos do fundo da enseada na expedição de novembro de 2004. Estas duas espécies encontram-se associadas, sendo difícil separação do tufo, por isso, optou-se por designá-las genericamente de Bryopsis spp. As algas foram removidas manualmente utilizando-se sacos plásticos para envolvê-las; posteriormente, foram acondicionadas em recipientes plásticos e fixadas em formol a 4\%. Foram coletados dados de temperatura da água, com termômetro graduado em escala Celsius em três dias de coletas no período diurno entre $9: 00$ às 12:00hrs e de salinidade da água, utilizando-se 
salinômetro. A profundidade do local da coleta foi medida com o auxílio de uma trena. Em laboratório, o processamento das amostras envolveu lavagens sucessivas das plantas em baldes plásticos, e retenção da fauna em peneiras com tela de "nylon" com $500 \mu \mathrm{m}$ de abertura de malha, comumente utilizada para estudos de macrofauna de fital.

A triagem foi realizada sob microscópio estereoscópico e a carcinofauna foi separada em grandes grupos. Posteriormente, os crustáceos Decapoda e Amphipoda, Isopoda e Tanaidacea foram identificados em nível específico, quando possível. Para cada espécie identificada são fornecidos a diagnose, material examinado, os dados ecológicos e a distribuição geográfica baseados em literatura especializada, seguido pelos comentários, quando necessário. Para os Táxons identificados em nível de gênero são citados a diagnose, material examinado e comentários, quando pertinente.

A abundancia relativa foi obtida por 100\% ( $x$ abundância da espécie / abundância total de todas as espécies). As espécies com abundância relativa inferior a $10 \%$ foram reunidas como "outros". A densidade é o número de indivíduos de cada espécie na composição da comunidade. A densidade absoluta foi obtida pela contagem do número de indivíduos amostrados de uma determinada espécie (ni) na área amostral (ha): DA = ni/ha.

O Material encontra-se depositado na coleção Carcinológica do Departamento de Oceanografia da Universidade Federal de Pernambuco. 


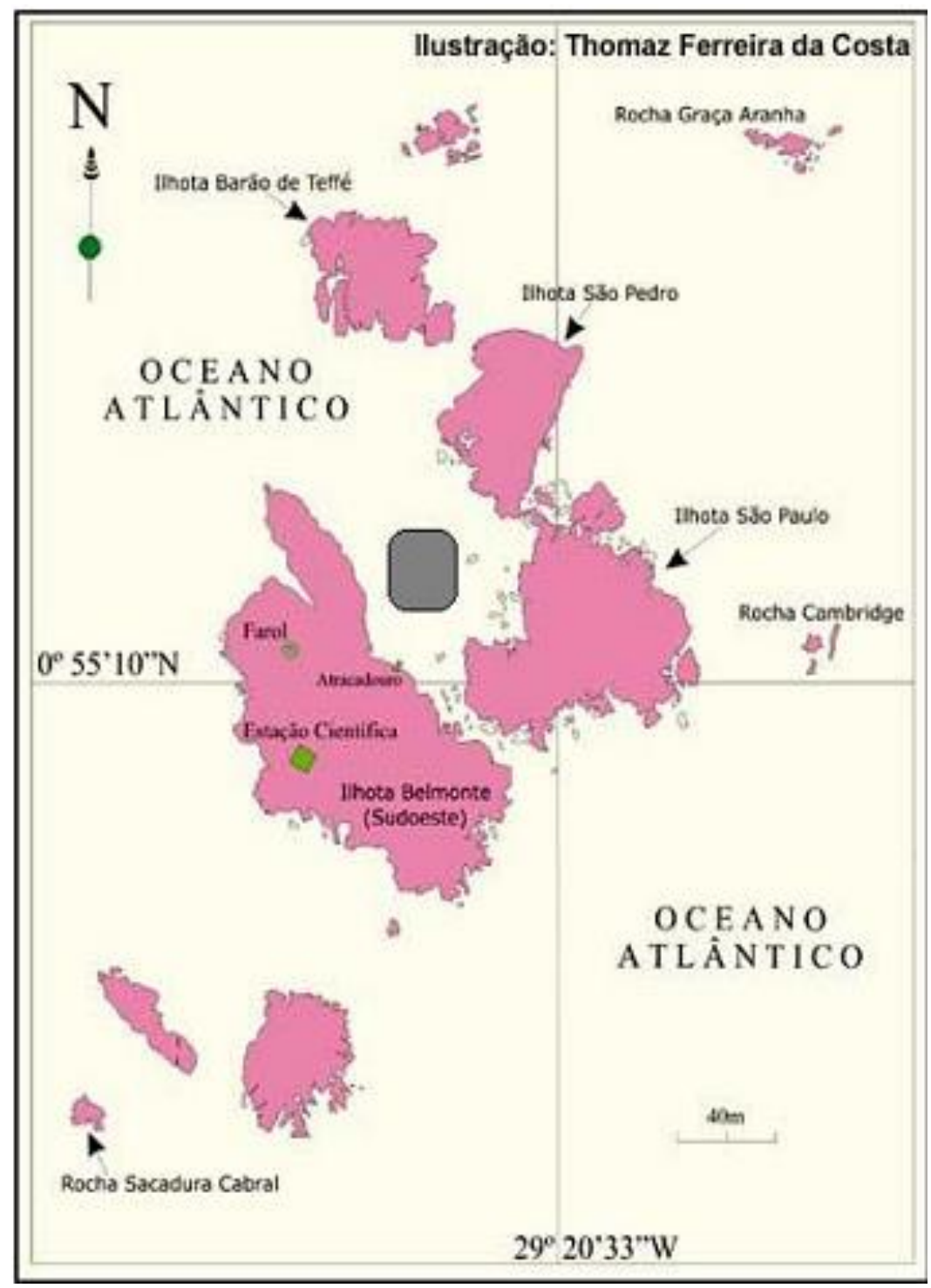

Figura 2. Arquipélago de São Pedro e São Paulo com indicação da enseada (estação fixa). Fonte: http://www.pdic.com.br/pdic2005/biblioteca/spsp.asp.

\section{RESULTADOS}

\section{DADOS ABIÓTICOS}

A salinidade da água da enseada apresentou valor de $37 \%$ em todas as amostras.

A temperatura da água variou entre $28^{\circ} \mathrm{C}$ e $32,2^{\circ} \mathrm{C}$.

\section{CARCINOFAUNA}

A carcinofauna do Arquipélago de São Pedro e São Paulo esteve representada por 4 Ordens, 12 Famílias, 15 Gêneros e 10 Espécies. Foram coletados 1662 exemplares, sendo 337 Tanaidacea, 451 Isopoda, 853 Amphipoda e 21 Decapoda (Tab. 1). 
Silva, Carcinofauna associada ao fital Caulerpa racemosa (Forsskal) J. Agardh e Bryopsis spp. do Arquipélago de São Pedro e São Paulo - Brasil.

Tabela 1. Número de indivíduos coletados de tanaidáceos, anfípodos, isópodos e decápodos associados a Caulerpa racemosa e Bryopsis spp. no Arquipélago de São Pedro e São Paulo (NE, Brasil), com profundidade de coleta $7 \mathrm{~m}$.

\begin{tabular}{|c|c|c|c|}
\hline CRUSTACEA & C. racemosa & Briopsis SP. & Profundidade \\
\hline Psamokalliapseudes granulosus & 239 & 25 & $7 m$ \\
\hline Calozodion sp. & 0 & 2 & $7 m$ \\
\hline Leptochelia dubia & 6 & 0 & $7 m$ \\
\hline Joeropsis sp. & 197 & 26 & $7 m$ \\
\hline Amphitoe $s p$. & 26 & 11 & $7 \mathrm{~m}$ \\
\hline Cymadusa sp. & 31 & 0 & $7 \mathrm{~m}$ \\
\hline Hyale $s p$. & 116 & 35 & $7 \mathrm{~m}$ \\
\hline Hyale macrodactyla & 160 & 34 & $7 \mathrm{~m}$ \\
\hline Elasmopus sp. & 57 & 0 & $7 m$ \\
\hline Elasmopus pectenicrus & 177 & 0 & $7 m$ \\
\hline Elasmopus rapax & 69 & 27 & $7 m$ \\
\hline Elasmopus spinidactylus & 5 & 0 & $7 \mathrm{~m}$ \\
\hline Elasmopus brasiliensis & 3 & 0 & $7 \mathrm{~m}$ \\
\hline Stenothoe $s p$. & 3 & 5 & $7 m$ \\
\hline Quadrimaera sp. & 1 & 0 & $7 \mathrm{~m}$ \\
\hline Synalpheus sp. & 1 & 0 & $7 m$ \\
\hline Mithraculus forceps & 3 & 3 & $7 m$ \\
\hline Plagusia depressa & 1 & 0 & $7 \mathrm{~m}$ \\
\hline Nanocassiope melanodactyla & 9 & 2 & $7 m$ \\
\hline TOTAL & 1104 & 170 & \\
\hline
\end{tabular}

Tabela 2. Número de indivíduos coletados de tanaidáceos, anfípodos, isópodos e decápodos associados a Caulerpa racemosa e Bryopsis spp. no Arquipélago de São Pedro e São Paulo (NE, Brasil), coletados na superfície.

\begin{tabular}{lcc}
\hline \multicolumn{1}{c}{ CRUSTACEA } & C. racemosa & Profundidade \\
\hline Psamokalliapseudes granulosus & 63 & Superfície \\
Calozodion $s p$. & 1 & Superfície \\
Pseudosphyrapus sp. & 1 & Superfície \\
Joeropsis sp. & 6 & Superfície \\
Amphitoe sp. & 4 & Superfície \\
Hyale sp. & 17 & Superfície \\
Hyale macrodactyla & 25 & Superfície \\
Elasmopus sp. & 19 & Superfície \\
Elasmopus rapax & 25 & Superfície \\
Elasmopus spinidactylus & 3 & Superfície \\
\hline
\end{tabular}


Silva, Carcinofauna associada ao fital Caulerpa racemosa (Forsskal) J. Agardh e Bryopsis spp. do Arquipélago de São Pedro e São Paulo - Brasil.

A carcinofauna associada à Caulerpa racemosa esteve representada por número de indivíduos de 4 ordens: Amphipoda, Isopoda, Tanaidacea e Decapoda (Fig. 3). De acordo com a abundância relativa o grupo dominante foi Amphipoda com 50\% dos indivíduos amostrados, seguido pelos Isopoda, com $28 \%$ e pelos Tanaidacea, com $21 \%$. A ordem Decapoda esteve representada por apenas $1 \%$ do total. Observa-se que $99 \%$ da carcinofauna esteve constituída por Crustacea Peracarida.

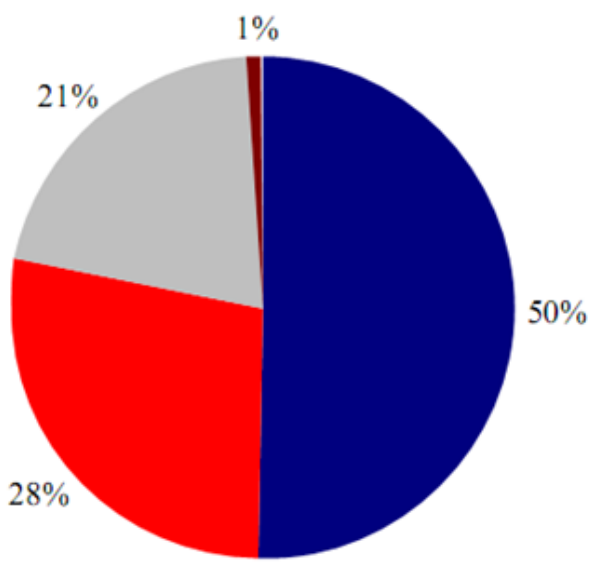

- Amphipoda Isopoda $\quad$ Tanaidacea Decapoda

Figura 3. Abundância relativa das ordens de crustáceos associadas a Caulerpa racemosa no Arquipélago de São Pedro e São Paulo coletadas nos dois gradientes de profundidade: superfície e $7 \mathrm{~m}$.

A carcinofauna associada à Bryopsis spp. esteve representada pelas ordens Amphipoda, Isopoda, Tanaidacea e Decapoda (Fig. 4). De acordo com a abundância relativa o grupo dominante foi Amphipoda com $62 \%$ dos indivíduos amostrados, seguido pelos Isopoda, com $20 \%$ e pelos Tanaidacea com 15\%. A ordem Decapoda contabilizou apenas 3\% do total, mostrando resultados semelhantes ao da carcinofauna associada à Caulerpa racemosa.

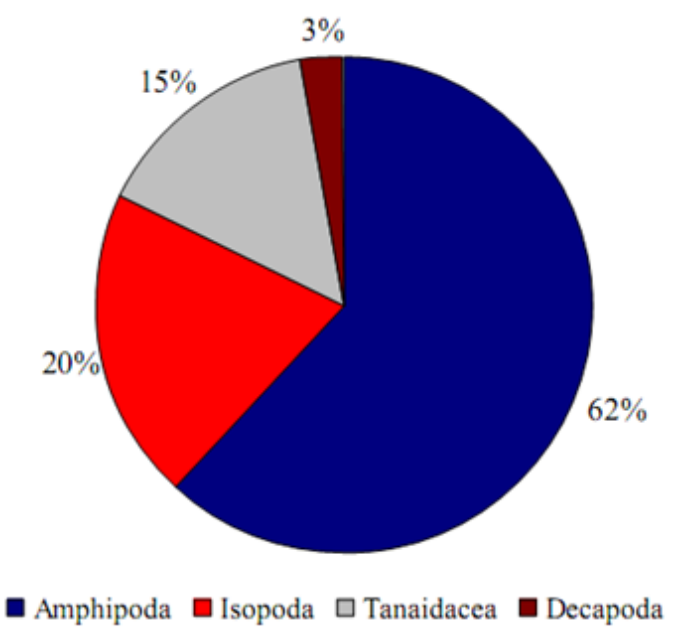

Figura 4. Abundância relativa das ordens de crustáceos associadas à Bryopsis spp. no Arquipélago de São Pedro e São Paulo coletada a 7 metros de profundidade. 
Silva, Carcinofauna associada ao fital Caulerpa racemosa (Forsskal) J. Agardh e Bryopsis spp. do Arquipélago de São Pedro e São Paulo - Brasil.

De acordo com a abundância relativa das espécies de crustáceos associadas à Caulerpa racemosa a espécie mais expressiva foi o Tanaidacea Psamokalliapseudes granulosus, com 19\% dos indivíduos amostrados, seguido pelos Isopoda Joeropsis sp.com 15\% dos espécimes e pelos Amphipoda Elasmopus pectenicrus com $14 \%$ e Hyale macrodactyla com $13 \%$ dos exemplares (Fig. 5). De acordo com a abundância relativa das espécies de Crustacea associadas à Bryopsis spp. as espécies mais bem representadas foram os Amphipoda Hyale sp. e Hyale macrodactyla, com 20\% dos exemplares cada, seguidos por Elasmopus rapax com $16 \%$ e pelos Tanaidacea Psamokalliapseudes granulosus com 15\% e o Isopoda Joeropsis sp. com 15\% (Fig. 6).

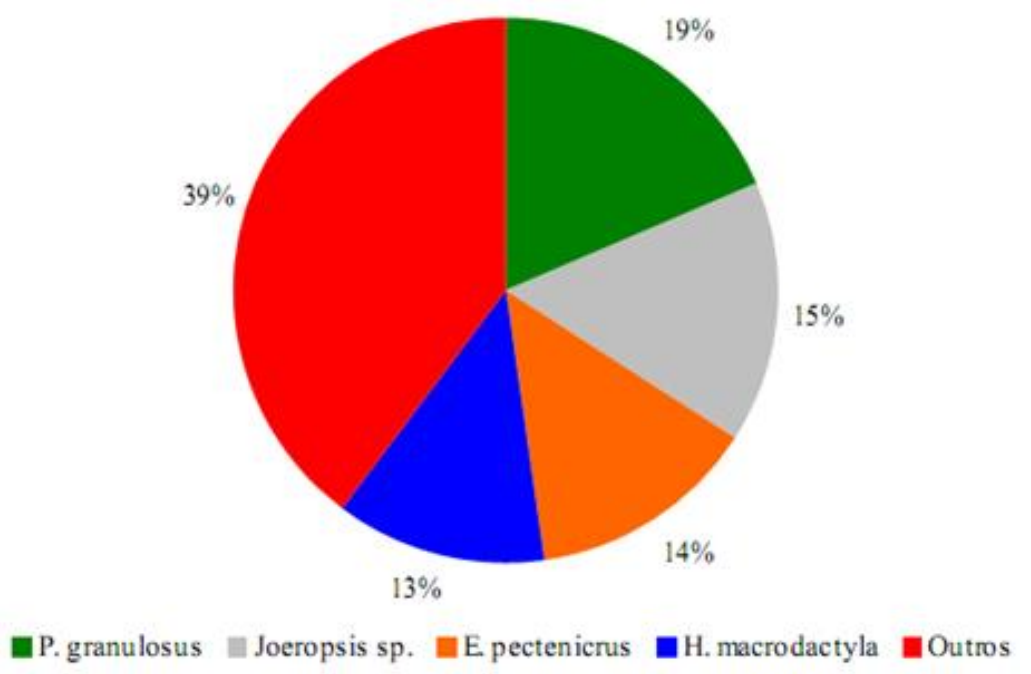

Figura 5. Abundância relativa das espécies de crustáceos associadas à Caulerpa racemosa no Arquipélago de São Pedro e São Paulo coletadas nos dois gradientes de profundidade: superfície e $7 \mathrm{~m}$.

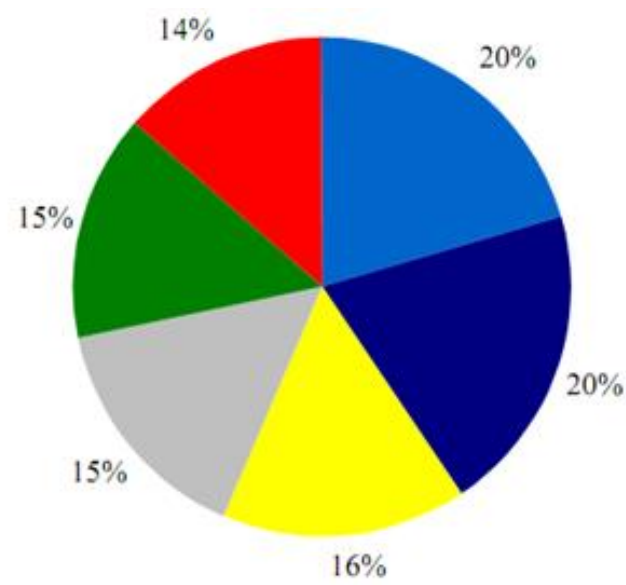

Hyale sp.

Elasmopus rapax

Psamokalliapseudes granulosus
Hyale macrodactyla Joeropsis sp. Outros

Figura 6. Abundância relativa das espécies de crustáceos associadas à Bryopsis spp. no Arquipélago São Pedro e São Paulo coletada a 7 metros de profundidade. 
Em Caulerpa racemosa as maiores densidades de indivíduos ocorreram no gradiente de profundidade de 7 metros. O Tanaidacea Psamokalliapseudes granulosus foi a espécie com maior densidade, apresentando um total de 239 espécimes, seguido pelo Isopoda Joeropsis sp. com 197 indivíduos, e os Amphipoda Elasmopus pectenicrus com 177, Hyale macrodactyla com 160, Hyale sp. com 116 e Elasmopus rapax com 69; as demais espécies de Crustacea identificados apresentaram densidades inferiores a 50 indivíduos. Nas amostras de superfície as densidades foram muito baixas; Psamokalliapseudes granulosus foi a espécie que apresentou a maior densidade populacional com 63 indivíduos, as demais espécies apresentaram densidades igual ou inferior a 25 indivíduos.

A carcinofauna associada à Bryopsis spp. coletada a 7 metros de profundidade apresentou baixa densidade de indivíduos. Hyale sp. apresentou a maior densidade, com 35 indivíduos, seguida por $H$. macrodactyla com 34 espécimes, Elasmopus rapax com 27, Joeropsis sp. com 26 indivíduos e $P$. granulosus com 25 exemplares. As espécies de Bryopsis foram mais colonizadas por Amphipoda, seguida pelos Isopoda e pelos Tanaidacea, o que reforça a teoria de dominância numérica de Crustacea Peracarida associados a algas moles. Contudo, quando comparada com as densidades de indivíduos encontradas em associação com Caulerpa racemosa, a fauna de crustáceos associados à alga Bryopsis spp. foi bem inferior, isto pode estar relacionado com a morfologia da fronde, que parece oferecer menor disponibilidade de microhabitats para os animais.

\section{Lista Taxonômica}

Filo Crustacea

Subfilo Malacostraca

Classe Caridoida

Subclasse Eucarida

Ordem Decapoda Dana, 1852

Infraordem Caridea Dana, 1852

Família Alpheidae Rafinesque, 1815

Gênero Synalpheus Bate, 1888

Material examinado: um exemplar juvenil em Caulerpa racemosa no fundo da enseada; XI. 2004; 7m.

Comentários: 0 indivíduo juvenil Synalpheus sp. (Figura 7), estava danificado e faltando alguns apêndices o que impossibilitou a identificação em nível de espécie. Holthuis et al. (1980) e Viana et al. (2004) registraram a presença de Synalpheus fritzmuelleri Coutière, 1909 no Arquipélago São Pedro e São Paulo. 


\section{Synalpheus sp}

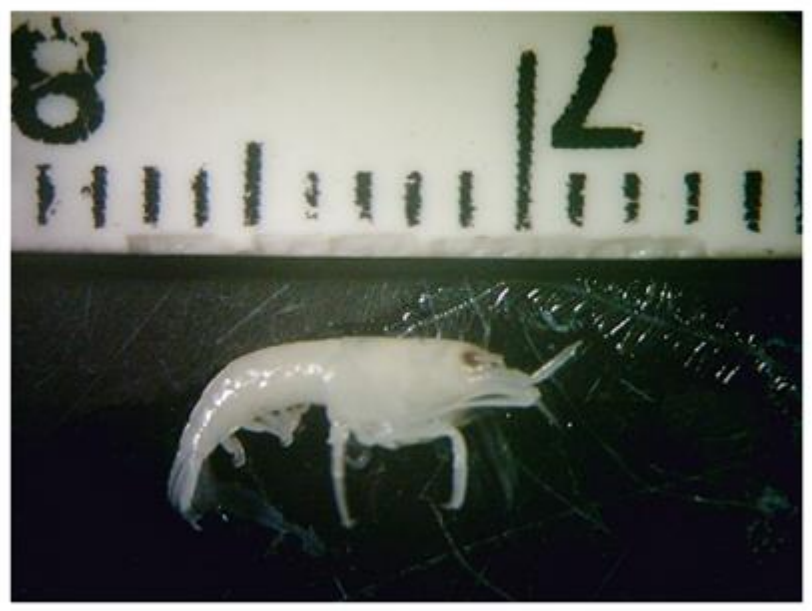

Figura 7. Vista lateral de Synalpheus sp.

Infraordem Brachyura Latreille, 1802

Seção Oxyrhyncha Latreille, 1803

Família Majidae Samouelle, 1819

Subfamília Mithracinae MacLeay,1838

Gênero Mithraculus White, 1847

Material examinado: Foram encontrados três exemplares machos de Mithraculus forceps (Figura 8), em Caulerpa racemosa no fundo da enseada, XI. 2004, 7m e 3 exemplares machos em Bryopsis spp., XI, 2004, 7m.

Distribuição Geográfica: Atlântico ocidental - da Carolina do Norte ao sul da Flórida, Golfo do México, Antilhas, Venezuela e Brasil (Fernando de Noronha e Rocas, e do Maranhão até São Paulo) (Melo, 1996); Arquipélago de Abrolhos (Holthuis et al., 1980); Rochedos São Pedro e São Paulo (Holthuis et al., 1980; Viana et al., 2004).

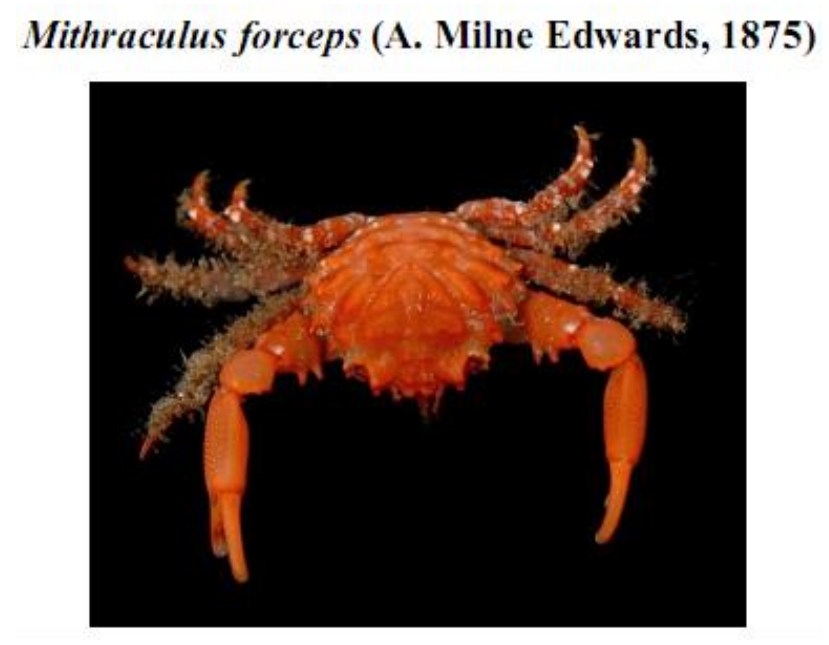

Figura 8. Vista dorsal de Mithraculus forceps.

Fonte:http://www.dnr.sc.gov/marine/sertc/Crustacean\%20gallery.htm 
Silva, Carcinofauna associada ao fital Caulerpa racemosa (Forsskal) J. Agardh e Bryopsis spp. do Arquipélago de São Pedro e São Paulo - Brasil.

Seção Brachyrhyncha Borradaile, 1907

Família Grapsidae Macleay, 1838

Subfamília Plagusiinae Dana, 1851

Gênero Plagusia Latreille, 1804

Material examinado: Encontrado um único exemplar macho Plagusia depressa (Figura 9), em Caulerpa racemosa, XI. 2004, 7m.

Dados ecológicos: Em fissuras das rochas e corais, poças de maré e no entremarés (Melo, 1996).

Distribuição geográfica: Atlântico Ocidental: Carolina do Norte e Carolina do Sul, Flórida, Golfo do México, Antilhas e Brasil (Fernando de Noronha, Rocas, Rochedos São Pedro e São Paulo, Trindade e do Ceará até a Bahia). Atlântico Oriental: Açores e Madeira, e do Senegal até Angola (Melo, 1996).

Plagusia depressa (Fabricius, 1775).

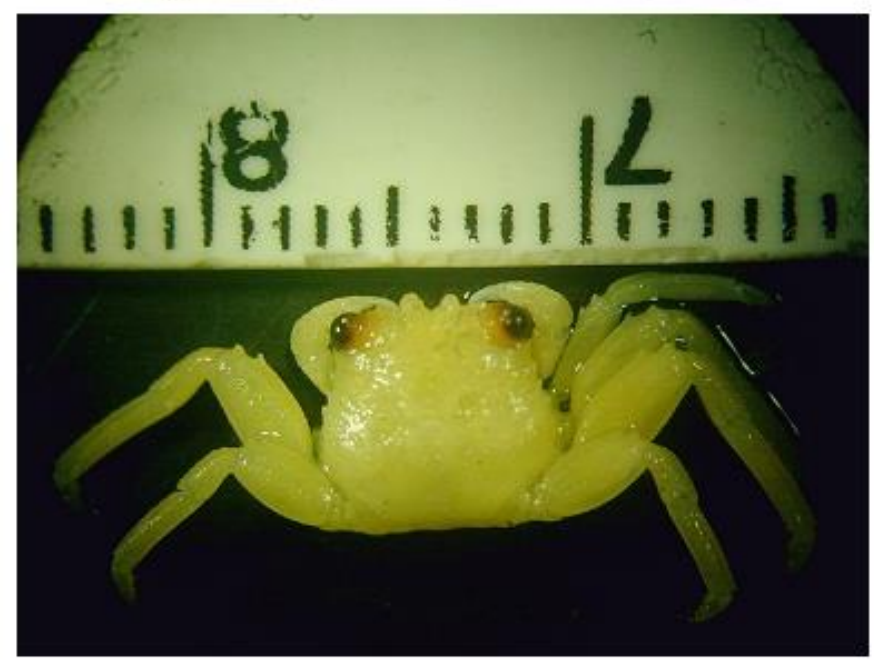

Figura 9. Vista dorsal Plagusia depressa.

Família Xanthidae MacLeay, 1838

Gênero Nanocassiope Guinot, 1967

Comentários: Espécie sublitoral, geralmente preferindo fundo com algas, corais e foraminíferos ou poças de areia. De 70 registros recentes da profundidade para a espécie fora da África Ocidental. 56 ou $80 \%$ são de profundidades de $50 \mathrm{~m}$ ou menos. Dois daqueles são da costa, o restante nas profundidades de 3-11 metros a 40-50m ou 9-69m dos 14 registros de uma água mais profunda, dois, $200 \mathrm{~m}$ e $225 \mathrm{~m}$, são das profundidades mais extremamente de $100 \mathrm{~m}$ e a escala do restante de 60 a 85m (Manning and Holthuis, 1981). 
Silva, Carcinofauna associada ao fital Caulerpa racemosa (Forsskal) J. Agardh e Bryopsis spp. do Arquipélago de São Pedro e São Paulo - Brasil.

Material Examinado: nove exemplares em Caulerpa racemosa, XI. 2004, 7m; dois em Caulerpa racemosa XI. 2004 superfície; dois em tufos de Bryopsis spp., XI. 2004, 7m. Todos machos.

Distribuição Geográfica: Pacifico Oriental: Baixa Califórnia, Ilha de Cocos e Galápagos; Atlântico Central: Ilha de Santa Helena e Ilha de Ascenção; Atlântico Oriental: Açores, Madeira, Ilhas Desertas, Ilhas Canárias, Ilhas de Cabo Verde, e Sul de Angola e Senegal, Golfo do Guiné, Príncipe e São Tomé (Manning and Holthuis, 1981).

Comentários: primeiro registro de Nanocassiope melanodactyla (Figura 10), para o Atlântico Ocidental (presente trabalho).

\section{Nanocassiope melanodactyla (A. Milne-Edwards, 1867)}

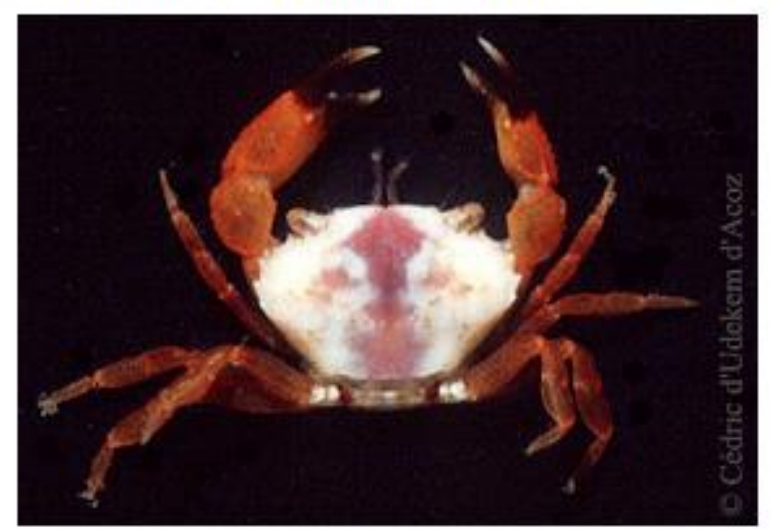

Figura 10. Vista dorsal de Nanocassiope melanodactyla.

Fonte:http://www.tmu.uit.no/crustikon/Decapoda/Decapoda2/Species_index/Nanocassiope_melanodacty. htm

Subfilo Malacostraca

Ordem Tanaidacea

Subordem Apseudomorpha Sieg, 1980

Família Kalliapseudidae Lang, 1956

Gênero Psammokalliapseudes Lang, 1956

Material examinado: 169 exemplares de Psammokalliapseudes granulosus (Figura 11), em C. racemosa de fundo em XI, 2004, 7m, 63 em superfície XI, 2004 e 25 em tufos de Bryopsis XI, 2004, 7m. 54 exemplares em $C$. racemosa no fundo V,2004, 7m. 16 em C. racemosa no fundo em I, 2003,7m.

Comentários: Atlântico Ocidental: Brasil (Ceará até Bahia). Primeiro registro para o Arquipélago de São Pedro e São Paulo (presente trabalho). 
Silva, Carcinofauna associada ao fital Caulerpa racemosa (Forsskal) J. Agardh e Bryopsis spp. do Arquipélago de São Pedro e São Paulo - Brasil.

\section{Psammokalliapseudes granulosus Silva-Brum, 1973}

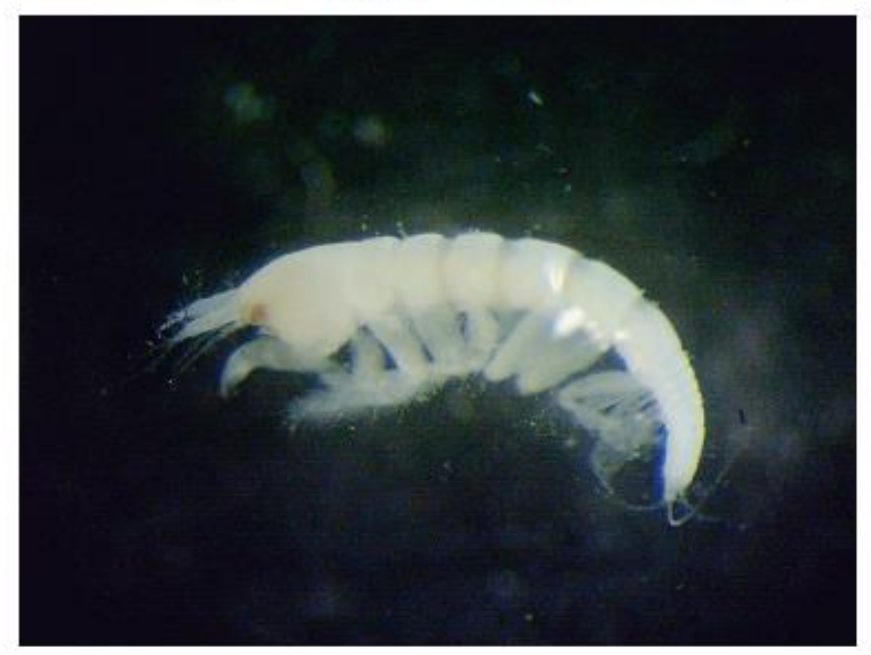

Figura 11. Vista lateral de Psammokalliapseudes granulosus.

Família Metapseudidae Lang, 1970

Gênero Calozodion Gardiner, 1973

Material examinado: um exemplar em C. racemosa em superfície XI, 2004 e dois em tufos de Bryopsis XI, 2004, 7m.

Comentários: Primeiro registro de Calozodion sp. (Figura 12), para o Atlântico Ocidental (presente trabalho).

\section{Calozodion sp.}

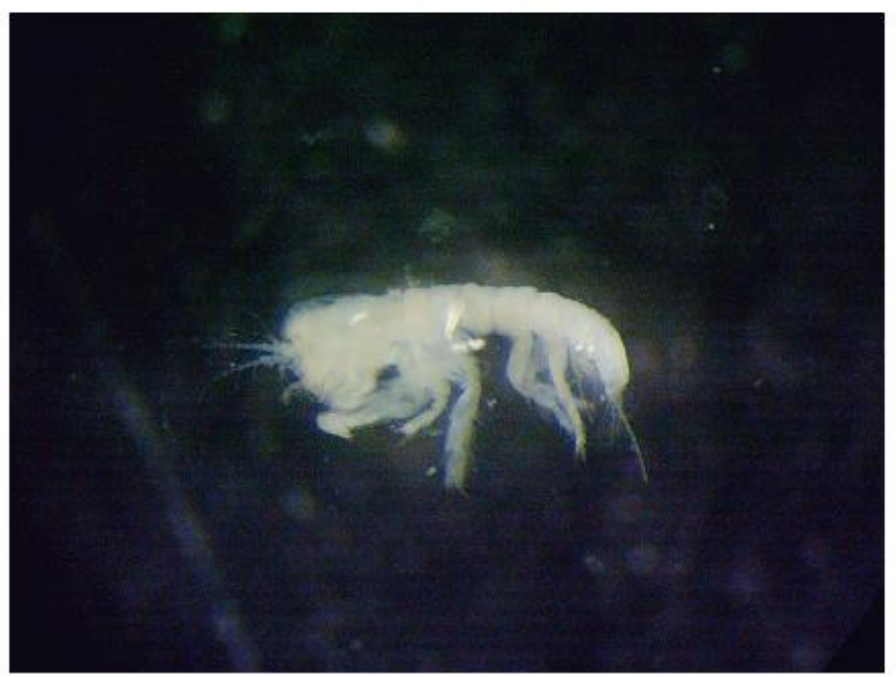

Figura 12. Vista lateral de Calozodion sp.

Subordem Tanaidomorpha Sieg, 1980

Família Leptocheliidae Lang, 1973

Gênero Leptochelia Dana, 1849 
Silva, Carcinofauna associada ao fital Caulerpa racemosa (Forsskal) J. Agardh e Bryopsis spp. do Arquipélago de São Pedro e São Paulo - Brasil.

Material examinado: dois exemplares de Leptochelia dubia (Figura 13), em C. racemosa no fundo XI, 2004, 7m. Um em C. racemosa no fundo em V, 2004, 7m e três em C. racemosa no fundo em I, 2003, $7 \mathrm{~m}$.

Distribuição Geográfica: Pacífico Ocidental, Oceano Índico, Mar Mediterrâneo, Canal de Suez, Mar Vermelho, Mar Negro, Atlântico Ocidental: Costa leste da América do Norte, Antilhas e Brasil (Maranhão até São Paulo). Arquipélago de São Pedro e São Paulo (presente trabalho).

\section{Leptochelia dubia (Kroyer, 1842)}

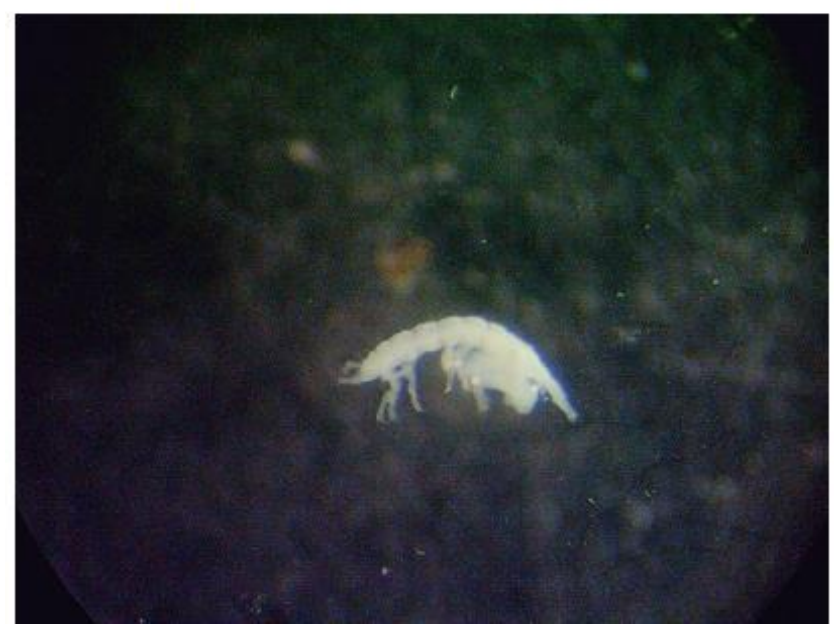

Figura 13. Vista lateral de Leptochelia dubia.

Pseudosphyrapus Gutu, 1980

Material examinado: um exemplar em C. racemosa na superfície em XI, 2004.

Comentários: Brasil - Rio de Janeiro. Primeiro registro de Pseudophyrapus sp. (Figura 14), para o Arquipélago de São Pedro e São Paulo (presente trabalho).

Pseudosphyrapus sp.

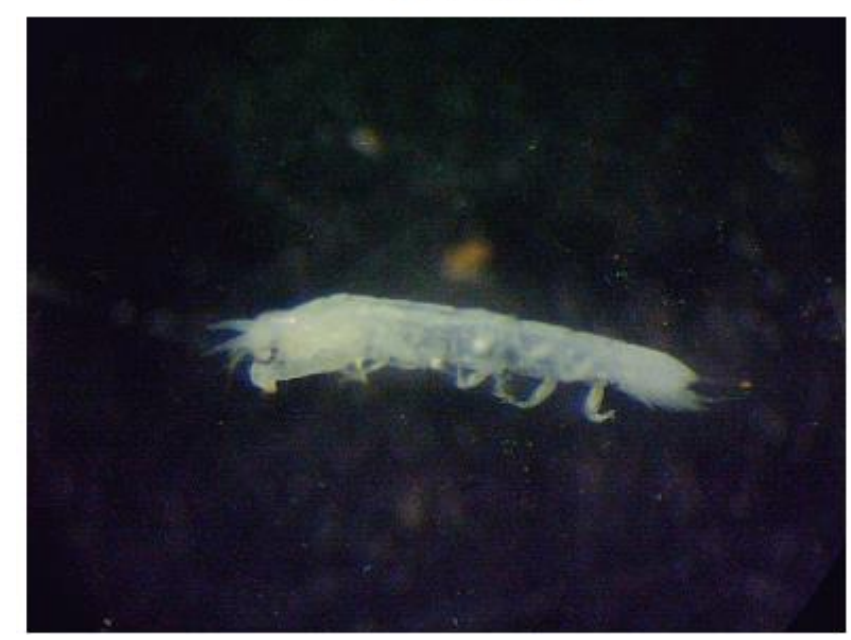

Figura 14. Vista lateral de Pseudosphyrapus sp. 
Silva, Carcinofauna associada ao fital Caulerpa racemosa (Forsskal) J. Agardh e Bryopsis spp. do Arquipélago de São Pedro e São Paulo - Brasil.

Ordem Amphipoda

Subordem Gammaridea Latreille, 1803

Família Amphithoidae Stebbing, 1899

Gênero Amphitoe Leach, 1814

Material Examinado: 26 exemplares de Amphitoe sp. (Figura 15) em Caulerpa racemosa no fundo, XI 2004, 7m, quatro em superfície XI, 2004 e 11 em Bryopsis spp., XI, 2004, 7m. Comentários: Gênero amplo cosmopolita (Lincoln, 1979). Primeiro registro do gênero para o Arquipélago de São Pedro e São Paulo (presente trabalho).

\section{Amphitoe sp.}

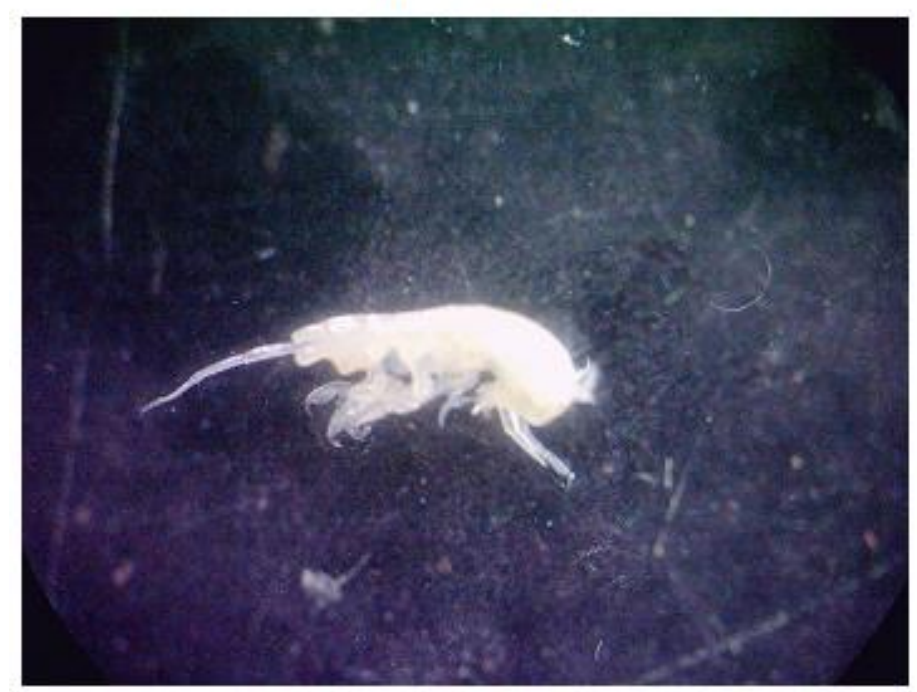

Figura 15. Vista lateral de Amphitoe sp.

Gênero Cymadusa Savigny, 1816

Material Examinado: 31 exemplares de Cymadusa sp. (Figura 16), em Caulerpa racemosa no fundo da enseada, XI 2004, $7 \mathrm{~m}$.

Comentários: Flórida, Golfo do México, Mar do Caribe, Oceano Atlântico, Pacífico e Índico, Mares do Mediterrâneo e Vermelho (Wakabara, 1972). Primeiro registro do gênero para o Arquipélago de São Pedro e São Paulo (presente trabalho). 


\section{Cymadusa sp.}

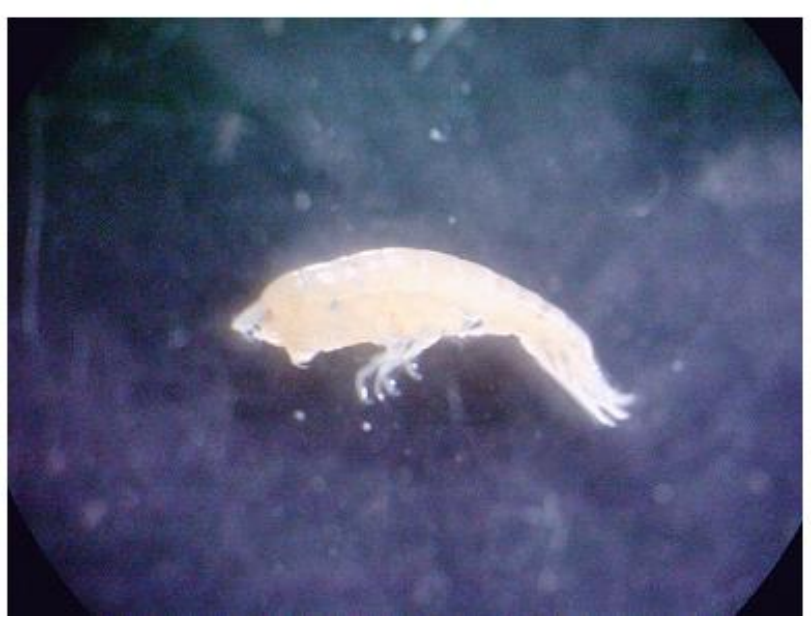

Figura 16. Vista lateral de Cymadusa sp.

Família Hyalidae Bulycheva, 1957

Gênero Hyale Rathke, 1837

Material Examinado: 46 exemplares de Hyale sp. (Figura 17) em Caulerpa racemosa, I, 2003, 7m; 33 em C. racemosa V.2004, 7m; 70 em C. racemosa XI, 2004, 7m e 17 em superfície XI, 2004. 11 em Bryopsis spp., XI, 2004, 7m.

Comentários: Cosmopolita, a maioria das espécies ocorre em áreas Tropicais (Lincoln, 1979). Primeiro registro do gênero para o Arquipélago de São Pedro e São Paulo (presente trabalho).

Gênero Hyale sp.

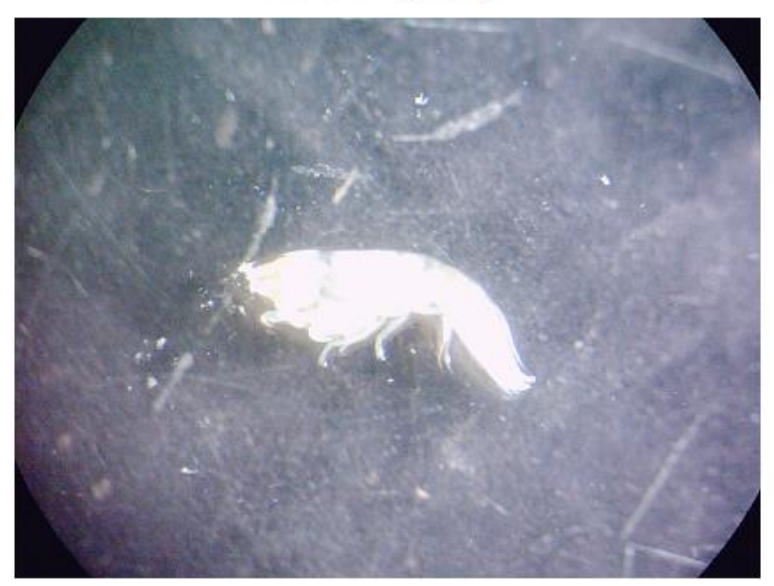

Figura 17. Vista lateral de Hyale sp.

Material Examinado: 54 exemplares de Hyale macrodactyla (Figura 18), em Caulerpa racemosa no fundo, V, 2004, 7m e 106 em C. racemosa XI, 2004, 7m. 25 exemplares na superfície, XI, 2004. E 34 em Bryopsis spp. XI, 2004, 7m.

Distribuição Geográfica: Oceano Atlântico, Brasil, RJ (YOUNG, 1998), Arquipélago de São Pedro e São Paulo (presente trabalho). 
Silva, Carcinofauna associada ao fital Caulerpa racemosa (Forsskal) J. Agardh e Bryopsis spp. do Arquipélago de São Pedro e São Paulo - Brasil.

\section{Hyale macrodactyla Stebbing, 1899}

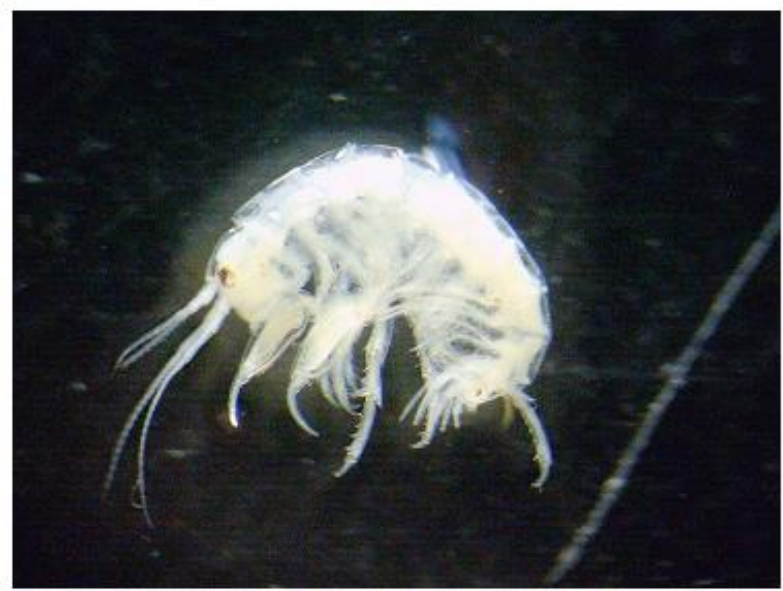

Figura 18. Vista lateral de Hyale macrodactyla.

Família Melitidae Bousfield, 1973

Gênero Elasmopus Costa, 1853

Material examinado: 57 exemplares de Elasmopus sp. (Figura 19), em Caulerpa racemosa, XI, 2004, 7m e 19 em C. racemosa superfície, XI, 2004.

Comentários: Cosmopolita (Serejo, 2000). Primeiro registro do gênero para o Arquipélago de São Pedro e São Paulo (presente trabalho).

\section{Elasmopus sp.}

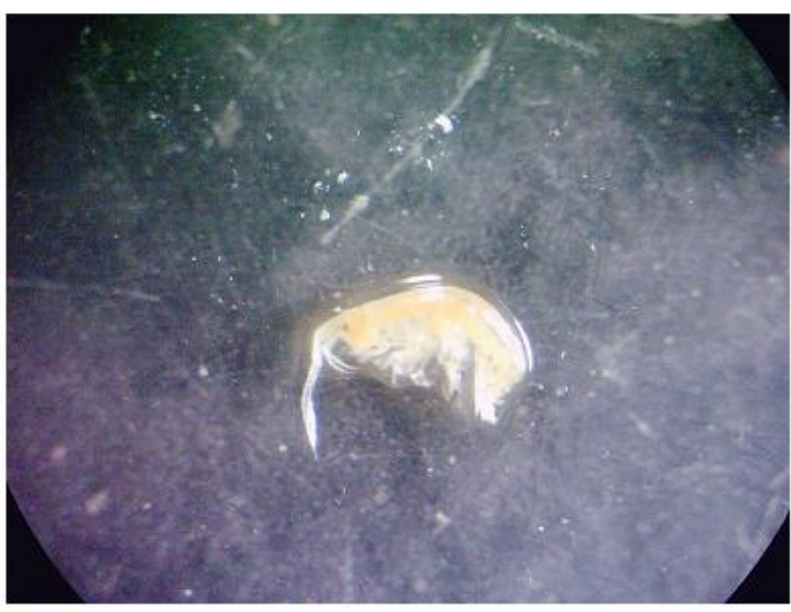

Figura 19. Vista lateral de Elasmopus sp.

Material examinado: 3 exemplares de Elasmopus brasiliensis (Figura 20), em C. racemosa no fundo XI, 2004, 7m.

Dados ecológicos: Ocorre desde a franja infralitoral até profundidades de 47-50m e pelos dados obtidos parece preferir algas como substrato (Wakabara, 1972). 
Silva, Carcinofauna associada ao fital Caulerpa racemosa (Forsskal) J. Agardh e Bryopsis spp. do Arquipélago de São Pedro e São Paulo - Brasil.

Distribuição Geográfica: Mediterrâneo, Atlântico, Pacífico e Caribe (Wakabara, 1972). Arquipélago de São Pedro e São Paulo (presente trabalho).

\section{Elasmopus brasiliensis (Dana, 1855)}

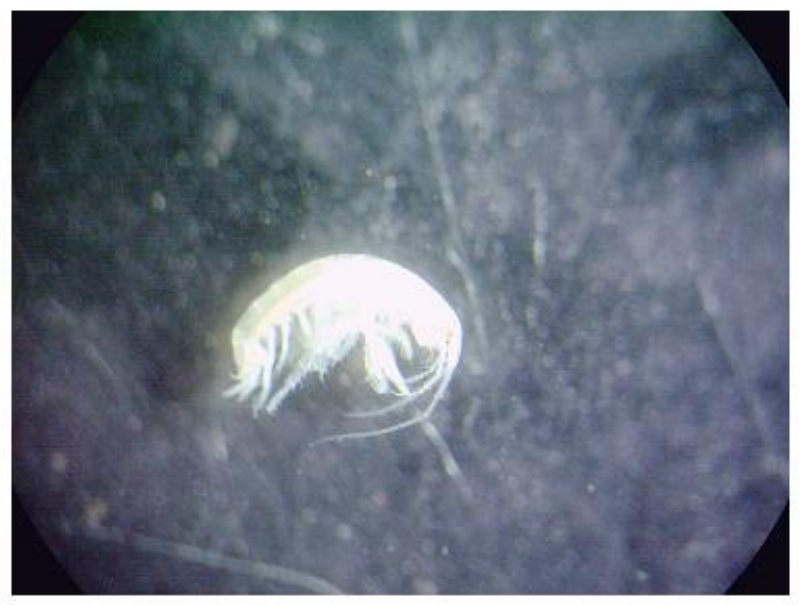

Figura 20. Vista lateral de Elasmopus brasiliensis.

Material examinado:74 exemplares de Elasmopus pectenicrus (Figura 21), em Caulerpa racemosa, I, 2003, 7m; e 103 em C. racemosa, XI, 2004, 7m. Dados ecológicos: Zona entremarés até a franja infralitoral e ocorrendo em algas (Wakabara, 1972).

Distribuição Geográfica: Em mares tropicais (Wakabara, 1972); Arquipélago de São Pedro e São Paulo (presente trabalho).

\section{Elasmopus pectenicrus (Bate, 1862)}

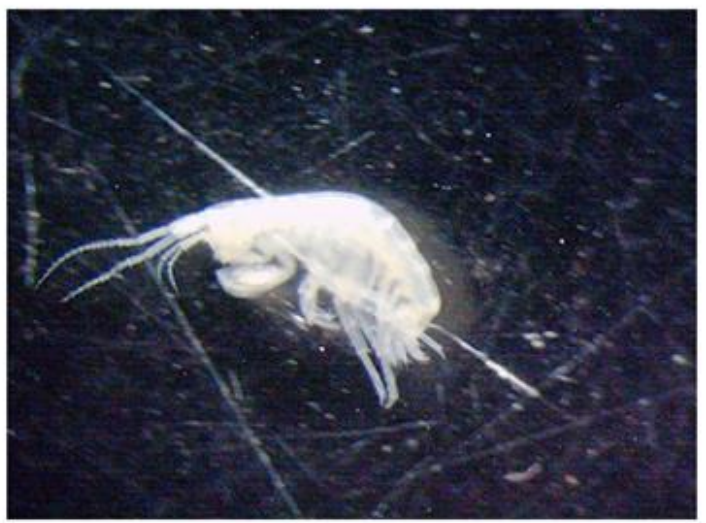

Figura 21. Vista lateral de Elasmopus pectenicrus.

Material examinado: 37 exemplares de Elasmopus rapax (Figura 22), em Caulerpa racemosa, I, 2003, 7m; 27 em tufos de Bryopsis spp., XI, 2004, 7m. 32 exemplares em C. racemosa XI, 2004, 7m e 25 em superfície XI, 2004. 
Silva, Carcinofauna associada ao fital Caulerpa racemosa (Forsskal) J. Agardh e Bryopsis spp. do Arquipélago de São Pedro e São Paulo - Brasil.

Dados ecológicos: Desde a franja infralitoral até profundidades até $42 \mathrm{~m}$, ocorrendo em algas (Wakabara, 1972).

Distribuição: Cosmopolita (Wakabara, 1972). Arquipélago de São Pedro e São Paulo (presente trabalho).

\section{Elasmopus rapax Costa, 1853}

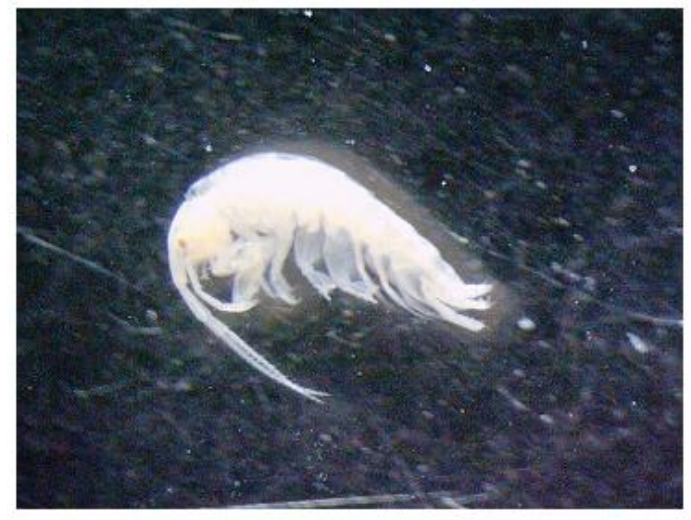

Figura 22. Vista lateral de Elasmopus rapax.

Material examinado: cinco exemplares de Elasmopus spinidactylus (Figura 23), em Caulerpa racemosa, XI 2004, 7m e três em superfície, XI, 2004.

Dados ecológicos: A espécie é habitante de águas rasas entre a zona entremarés e a franja infralitoral, e comum em algas. (Wakabara, 1972).

Distribuição Geográfica: Madagascar, Ilhas Maurícios, Arquipélago Chagos, Índia, Fiji, Micronésia, Polinésia, Hawaii, Venezuela (Apadoo and Myers, 2003). Pacífico e Atlântico Chagos Island - para este através de Micronésia, Hawaii, Clipperton Island SE Polinésia e Caribe - Venezuela (Barnard, 1970). Arquipélago de São Pedro e São Paulo (presente trabalho).

Elasmopus spinidactylus Chevreux, 1907

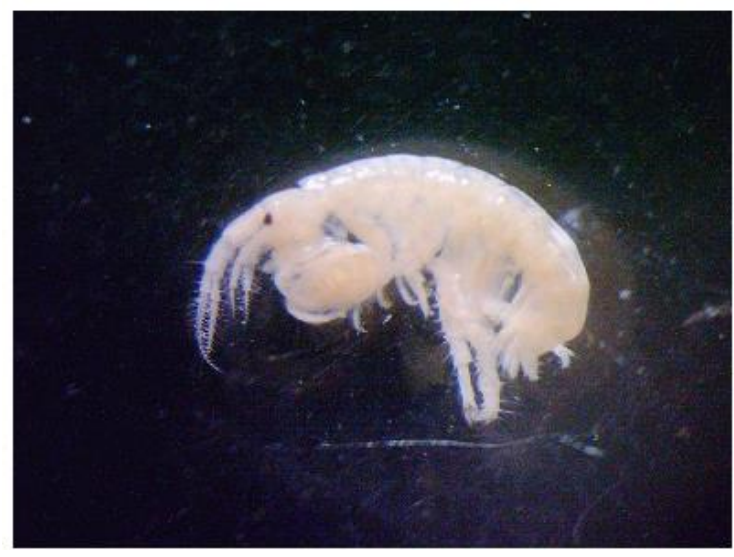

Figura 23. Vista lateral de Elasmopus spinidactylus. 
Silva, Carcinofauna associada ao fital Caulerpa racemosa (Forsskal) J. Agardh e Bryopsis spp. do Arquipélago de São Pedro e São Paulo - Brasil.

Gênero Quadrimaera Krapp-Schickel \& Ruffo, 2000

Material examinado: 1 exemplar de Quadrimaera sp. (Figura 24), em C. racemosa de fundo XI, 2004, 7m.

Comentários: larga distribuição, maioria zona tropical litoral. Com cerdas de 35 espécies conhecidas (Lincoln, 1979). Primeiro registro do gênero para o Arquipélago de São Pedro e São Paulo (presente trabalho).

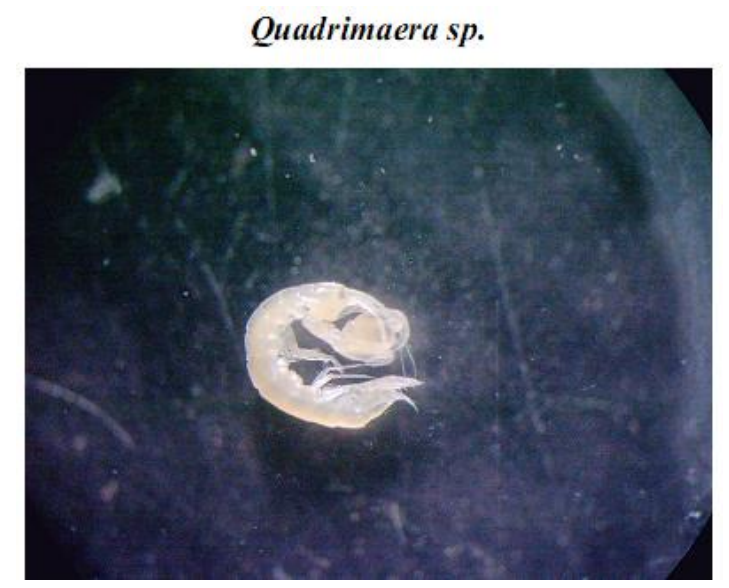

Figura 24. Vista lateral de Quadrimaera sp.

Família Stenothoidae Boeck, 1871

Gênero Stenothoe Dana, 1852

Material examinado: cinco exemplares de Stenothoe sp. (Figura 25), em tufos de Bryopsis spp., XI 2004, 7m. e três em C. racemosa XI, 2004, 7m.

Comentários: Cosmopolita (Lincoln, 1979). Primeiro registro do gênero para o Arquipélago de São Pedro e São Paulo (presente trabalho).

Stenothoe sp.

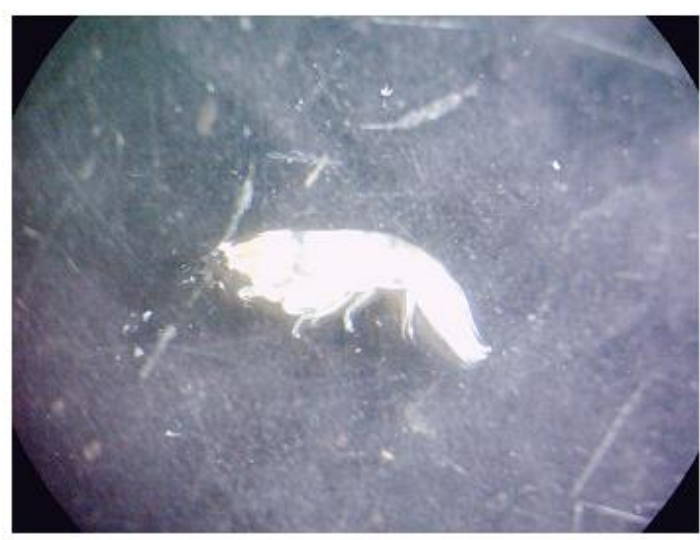

Figura 25. Vista lateral de Stenothoe sp. 
Silva, Carcinofauna associada ao fital Caulerpa racemosa (Forsskal) J. Agardh e Bryopsis spp. do Arquipélago de São Pedro e São Paulo - Brasil.

\section{Ordem Isopoda}

Suborder Asellota Latreille, 1803

Família Joeropsididae Nordenstam, 1933

Gênero Joeropsis Koehler, 1885

Diagnose: Olhos dorsoventrais presentes. Artigo da Antena basal maior e mais longo, frequentemente com dentição distal transparente. Antena, artigo peduncular, 3-5 um pouco dilatados, artigo 2 frequentemente com franja de escalas transparentes; flagelados aproximadamente com 6 artigos, mais próximo que o pedúnculo do artigo 5. Pereonitos similares, geralmente menor no comprimento e na largura. Urópodo geralmente com o ângulo mesodistal agudo; ramo reduzido (Kensley \& Schotte, 1989).

Material examinado: 135 exemplares de Joeropsis sp. (Figura 26), em C. racemosa no fundo em XI, 2004, 7m, 6 exemplares em superfície XI, 2004 e 26 em tufos de Bryopsis, XI, 2004. 54 exemplares em C. racemosa V, 2004, 7m e 8 exemplares em C. racemosa I, 2003, 7m.

Comentários: Cosmopolita. Primeiro registro do gênero para o Arquipélago de São Pedro e São Paulo (presente trabalho).

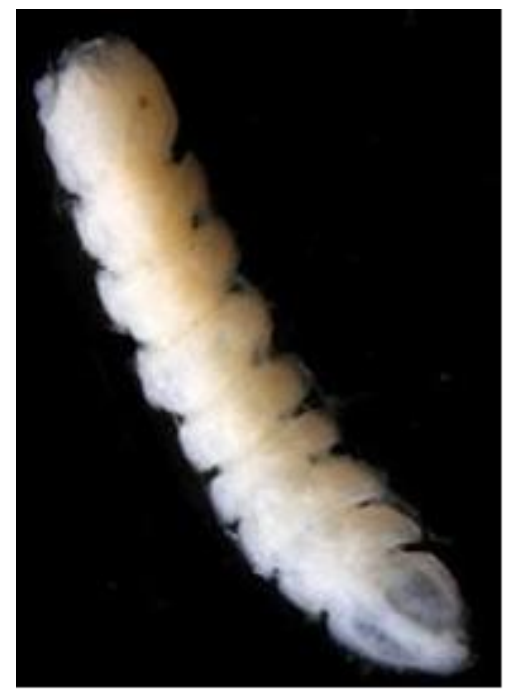

Figura 26. Visão Dorsal Joeropsis sp.

\section{DISCUSSÃO}

O Arquipélago de São Pedro e São Paulo possui um registro de 32 espécies de crustáceos devido aos poucos trabalhos desenvolvidos num local tão isolado; com o presente trabalho, cujo objetivo principal foi levantar os crustáceos associados aos fitais de macroalgas do ASPSP, essa lista aumenta para 40 espécies, com o registro de mais oito novas ocorrências de espécies e dez novos registros de gêneros que não foram possíveis de identificar em nível específico. As espécies de Amphipoda, Isopoda e Tanaidacea citadas são registradas pela primeira vez para o Arquipélago. A Ordem Amphipoda teve presença expressiva nos ecossistemas estudados. Em 
Silva, Carcinofauna associada ao fital Caulerpa racemosa (Forsskal) J. Agardh e Bryopsis spp. do Arquipélago de São Pedro e São Paulo - Brasil.

vários trabalhos sobre comunidade fital estes animais também estiveram bem representados, como para o fital Sargassum cymosum no litoral paulista (Tararam, 1977; Tararam and Wakabara, 1981), cujo grupo apresentou elevada participação numérica em grande parte do período estudado. Da mesma forma, no trabalho de Dutra (1985) a Ordem Amphipoda também teve número considerável nas amostras para o fital Pterocladia capillacea na Ilha do Mel (SP). Santos and Correia (1995) registraram números expressivos para os táxons Amphipoda e Decapoda do fital Halimeda coletados nos recifes de coral de Ponta Verde (AL). Trabalhos de Leite et al. (2003) relatam a presença de anfípodos gamarídeos ampitoideos associados às algas, principalmente Sargassum cymosum, com presença das espécies Amphitoe ramondi Audoin, 1826, Cymadusa filosa Savigny, 1816, Sunamphitoe pelagica Milne-Edwards, 1830. Os Gamarídeos ampitoideos constituem uma das famílias de anfípodos mais abundantes e comuns entre as associadas às algas. Apadoo and Myers (2003) registram espécie de Elasmopus pectenicrus em algas como Centroceras clavulatum, Sargassum sp., Ulva reticulata, Digenia simplex e E. spinidactylus em Acanthophora spicifera, Amansia glomerata, Centrocera clavulatum, Digenia simplex, Sargassum sp., Ulva lactuca. Neste trabalho ocorreram $E$. pectenicrus em Caulerpa racemosa na superfície e no fundo da enseada e E. spinidactylus em Caulerpa racemosa no fundo da enseada.

O fital Caulerpa racemosa possui uma fauna associada mais rica, abrigando maior número de espécies que o fital Bryopsis spp., tendo em vista que os anfípodos $E$. pectenicrus, $E$. spinidactylus e Cymadusa sp., e os decápodos $P$. depressa e Synalpheus sp. só ocorreram em C. racemosa. Este fato pode estar associado à própria morfologia da planta, que é mais ramificada do que Bryopsis e por ser extremamente dominante no arquipélago.

A fauna associada também teve diferença em relação à profundidade do fital, tendo em vista que as amostras retiradas no fundo da enseada foram mais significativas em relação ao número de indivíduos que as amostras coletadas próximo à superfície, onde ocorreram apenas os anfípodos E. spinidactylus, E. pectenicrus, Hyale macrodactyla, Hyale sp. e Elasmopus sp. Um dos fatores mais importantes é que no neste local o fital fica mais exposto à ação das ondas e ao dessecamento, durante o período de maré baixa. Nesta fase, os organismos tendem a se proteger por baixo das frondes das algas, ou mesmo a migrar para regiões submersas do fital. Foi registrada no presente trabalho a primeira ocorrência da espécie africana Nanocassiope melanodactyla.

O Arquipélago de São Pedro e São Paulo situa-se no meio do Oceano Atlântico entre os continentes Sul-americano e Africano e recebe influência da corrente marítima Sul Equatorial no sentido Leste-Oeste e da contracorrente Sul Equatorial em sentido contrário, a qual situa-se de 60 a 100m de profundidade abaixo da primeira (Travassos et al., 1999). Os demais animais em grande parte possuem uma distribuição cosmopolita e registros para o Oceano Atlântico. 


\section{CONCLUSÃO}

Todos os táxons de crustáceos peracáridos coletados pertencentes aos grupos dos anfípodos (Amphitoe sp., Cymadusa sp., Hyale sp., Hyale macrodactyla, Elasmopus sp., Elasmopus pectenicrus, Elasmopus rapax, E. spinidactylus, E. brasiliensis, Stenothoe sp. e Quadrimaera sp.), tanaidáceos (Psamokalliapseudes granulosus, Calozodion sp., Leptochelia dubia e Pseudosphyrapus sp.) e isópodos (Joeropsis sp.) são referidos pela primeira vez para o Arquipélago de São Pedro e São Paulo, demonstrando a importância de estudos em ambiente de fital de ilhas. O maior número de indivíduos encontrados nas amostras de fundo ( $7 \mathrm{~m})$ sugerem que as plantas abrigam mais animais onde não sofrem ação direta das ondas, fornecendo maior abrigo e proteção. O maior número de espécies e espécimes de crustáceos associados à Caulerpa racemosa em relação aos tufos de Bryopsis spp. pode estar relacionado com a morfologia da fronde da primeira, que por ser mais ramificada oferece maior disponibilidade de microhabitats para estes animais, além desta ter sido encontrada em maior quantidade no ambiente.

O Xantídeo Nanocassiope melanodactyla tem seu primeiro registro para o Atlântico ocidental, sugerindo a importância das ilhas oceânicas como pontos intermediários para a migração de espécies de uma para a outra margem do Oceano Atlântico.

\section{AGRADECIMENTOS}

Ao meu Orientador Dr. Petrônio Alves Coelho pela ajuda e por sempre estar disposto com paciência e amizade, ao professor Dr. Marcos Souto Alves pela ajuda e principalmente pela amizade em todas as horas, aos amigos de Laboratório Catarina Silva e Jesser Souza-Filho pela identificação dos crustáceos anfípodos e tanaidáceos; ao amigo Douglas Burgos pela identificação das algas e pela ajuda, à secretaria do Departamento de Oceanografia, à Marinha do Brasil e ao Programa Arquipélago por tornar possível a realização deste trabalho, aos pescadores do Transmar I e II pela ajuda e amizade, ao CNPq - Conselho Nacional de Desenvolvimento Científico e Tecnológico pela concessão de Bolsa durante o curso.

\section{REFERÊNCIAS BIBLIOGRÁFICAS}

Alves, M.S. (1991), Macrofauna do fital Halodule wrightii Aschers (Angiospermae Potamogetonaceae) da Praia de Jaguaribe - Ilha de Itamaracá, Pernambuco-Brasil, Dissertação de Mestrado, Programa de Pós-Graduação em Oceanografia, Universidade Federal de Pernambuco, 315p. 
Silva, Carcinofauna associada ao fital Caulerpa racemosa (Forsskal) J. Agardh e Bryopsis spp. do Arquipélago de São Pedro e São Paulo - Brasil.

Apadoo, C.E. and Myers, A.A. (2003), The Genus Elasmopus (Crustacea: Amphipoda: Melitidae) From Mauritius (Indian Ocean) With Description Of Five New Species, Records of the Australian Museum, Vol. 55, pp. 61-84.

Barnard, J.L. (1970), Sublittoral Gammaridea (Amphipoda) of the Hawaiian Islands, Smithsonian Contributions to Zoology, Vol. 34, pp. 1- 286.

Dutra, R.R.C. (1985), A Fauna Vágil do Fital Pterocladia capillacea (Gmelin) Bornet \& Turet (Rhodophyta, Gelidiaceae) da Ilha do Mel, Paranaguá, Paraná, Tese de Mestrado, Universidade Federal do Paraná, 115p.

Friedrich, H. (1964), Marine Biology, Sidgwick \& Jackson Ltda, 467p.

Hargeman, L. (1966), The macro- and microfauna associated with Fucus serratus L., with some ecological remarks, Ophelia, Vol.3, pp. 1-3.

Holthuis, L.B., Edwards, A.J. and Lubbock, H.R. (1980), The Decapod and Stomatopod Crustacea of St Paul's Rocks, Zoologische Mededelingen, Vol.56, n. 3, pp. 27-51.

Leite, F.P.P. and Guth, A.Z. (2003), Morphological variations of post-marsupial stages of Sunampithoe pelagica Milne-Edwards (Crustacea, Amphipoda, Gammaridea, Ampithoidae) associated to Sargassum cymosum C. Agardh, Revista Brasileira de Zoologia, Vol.20, n.1, p.65-73.

Lincoln, R.J. (1979), British Marine Amphipoda: Gammaridea, British Museum (Natural History), $658 p$.

Manning, R.B. and Holthuis, L.B. (1981), West African Brachyuran Crabs (Crustacea: Decapoda), Smithsonian Institution Press, 379 p.

Melo, G.A.S. (1996), Manual de identificação dos Brachyura (Caranguejos e Siris) do litoral brasileiro, São Paulo: Plêiade/ Fapesp, 604p.

Santos, C.G. and Correia, M.D. (1995), Fauna associada ao fital Halimeda opuntia (Linnaeus) Lamouroux (Chlorophyta) do recife da Ponta Verde, Maceió, Alagoas, Brasil, Revista Brasileira de Zoologia, Vol. 12, n. 2, pp. 263-271.

Serejo, C.S. (2000), A New species of Amphipoda from the Brazilian Coast, with redescription of HyalespinidactylaChevreux, 1925 (Crustacea, Amphipoda, Hyalidae), Zoosystema, Vol. 23, n. 3, pp. 479-492.

Tararam, A.S. (1977), A Fauna Vágil de Sargassum cymosum C. Agardh, 1820 da Praia do Lamberto e Praia Grande, Ubatuba, São Paulo, com Especial Referência aos Gammaridea (Crustacea-Amphipoda), Dissertação de Mestrado, Universidade de São Paulo, 73p.

Tararam, A.S. and Wakabara, Y. (1981), The mobile fauna especially Gammaridea of Sargassum cymosum, Marine Ecology Progress Series, Vol. 5, pp. 157- 163. 
Silva, Carcinofauna associada ao fital Caulerpa racemosa (Forsskal) J. Agardh e Bryopsis spp. do Arquipélago de São Pedro e São Paulo - Brasil.

Travassos, P., Hazin, F.H.V., Zagaglia, J.R., Advincula, R. and Schober, J. (1999), Thermohaline structure around seamounts and islands off north-eastern Brazil, Archive of Fishery Marine Research, Vol. 47, pp. 211-222.

Viana, G.F.S, Ramos-Porto, M. and Torres, M.F.A. (2004), Crustáceos Decápodos coletados no Arquipélago de São Pedro e São Paulo, Brasil, Boletim Técnico-Científico do CEPENE, Vol. 12 , n. 1 , pp. $43-50$.

Wakabara, Y. (1972), Espécies da Família Gammaridae (Crustacea: Amphipoda) entre as latitudes $03^{\circ} 23^{\prime}$ e 38005'do Atlântico Ocidental, Tese de Doutorado, Universidade de São Paulo, 87p.

Young, P.S. (1998), Catalogue of Crustacea of Brazil, Museu Nacional/ UFR, Série Livros 7, $718 p$. 